\title{
Therapie bei Straffälligkeit: Zur Entwicklung der Sozialtherapie im deutschen Justizvollzug
}

\author{
Sonja Etzler ${ }^{1,2} \cdot$ Matthias Moosburner $^{1} \cdot$ Martin Rettenberger $^{1,3}$ \\ Eingegangen: 13. November 2019/ Angenommen: 19. Dezember 2019 / Online publiziert: 10. Februar 2020 \\ (c) Der/die Autor(en) 2020
}

\section{Zusammenfassung}

Die Stichtagserhebung der Kriminologischen Zentralstelle (KrimZ) fragt jedes Jahr zum Stichtag am 31. März die Gegebenheiten in allen sozialtherapeutischen Einrichtungen deutschlandweit ab. Inzwischen liegen Daten aus 23 Erhebungsjahren vor und geben Aufschluss über die Entwicklungen der Versorgungslage (Anzahl der Einrichtungen bzw. Haftplätze), bezüglich der demografischen Daten der Gefangenen (Alter, Staatsbürgerschaft, Dauer der Haftstrafe, schwerste Straftat, Vorstrafen), über institutionelle Vorgänge (Aufnahmen, Abgänge und Nachbetreuung) sowie hinsichtlich von Daten zum Personal (Anzahl der Personalstellen und Frauenanteil). Die vorliegenden Auswertungen verdeutlichen die Entwicklungstrends in der Sozialtherapie zwischen 1997 und 2019 und legen nahe, dass nach einem starken Ausbau der sozialtherapeutischen Einrichtungen ab 1969 nun mit 71 Einrichtungen eine Sättigungsgrenze erreicht zu sein scheint. Die inhaftierten Personen werden zunehmend älter, sodass 2019 die über 50-Jährigen die größte Altersgruppe stellen. Schon seit 2003 liegt der Anteil derjenigen, die aufgrund eines Sexualdelikts inhaftiert sind, bei ca. 50\%, was gegenüber anderen Deliktgruppen eine deutliche Mehrheit darstellt. Ein Großteil der Gefangenen hat keine Haftlockerungen, wobei hier eine zunehmend restriktivere Praxis zu erkennen ist. Die Personalausstattung hat sich über die letzten 23 Jahre insofern verändert, als dass mehr Fachdienste und tendenziell weniger Stellen im allgemeinen Vollzugsdienst (AVD) eingerichtet wurden.

Schlüsselwörter Längsschnitt · Vollerhebung $\cdot$ Stichtagserhebung $\cdot$ Behandlungsvollzug $\cdot$ Sexualstraftäter

\section{Treatment and delinquency: The development of social therapy units in the German prison system}

\begin{abstract}
The Centre for Criminology (Kriminologische Zentralstelle [KrimZ]) in Wiesbaden conducts an annual survey that records the data on 31 March from all social therapy units (STU) in the German prison system. In the meantime, longitudinal data derived from 23 years have been collected, which provide information about the supply situation (number and capacities of STUs), with respect to the demographic data of the inmates (age, citizenship, length of sentence, offenses, prior convictions), institutional processes (admissions, releases, aftercare) as well as data concerning the prison and treatment staff (number of positions and proportion of women). The current analyses clarify the developing trends of all German STUs from 1997 to 2019 and suggest that after a large expansion starting in 1969, a saturation limit has been reached with 71 STUs. The inmates in STUs tend to be older in age so that individuals aged 50 years or older are nowadays the largest group. Since 2003 around 50\% of inmates have been convicted of sexual offenses, which represents a clear majority compared to other offense groups. Furthermore, most of the offenders did not receive parole and this proportion has been growing during the last several years. Personnel resources changed within the last 23 years insofar as there are more special (treatment) services available (psychologists etc.) and at the same time less positions for prison officers.
\end{abstract}

Keywords Longitudinal study $\cdot$ Complete survey $\cdot$ Offender treatment $\cdot$ Sexual offenders $\cdot$ Law enforcement

Dr. Sonja Etzler

s.etzler@krimz.de

1 Kriminologische Zentralstelle (KrimZ), Wiesbaden, Deutschland
2 Goethe-Universität, Frankfurt am Main, Deutschland

3 Psychologisches Institut, Johannes Gutenberg-Universität, Mainz, Deutschland 
Im Rahmen der umfangreichen Strafrechtsreform im Jahr 1969 wurde in der Bundesrepublik Deutschland gemäß dem Resozialisierungsgedanken die Behandlung von straffällig gewordenen Personen zur Verringerung ihrer Rückfallwahrscheinlichkeit in den Mittelpunkt gerückt. Die Sozialtherapie im Strafvollzug wurde dabei als Maßregel zur Besserung und Sicherung mittels $\S 65$ des Strafgesetzbuchs (StGB) umgesetzt, neben den Maßregeln zur Unterbringung in einer psychiatrischen Klinik ( $\$ 63$ StGB) oder einer Entziehungsanstalt ( $\$ 64$ StGB): „Sozialtherapeutische Einrichtungen sollen es Menschen, die wegen erheblichen oder wiederholten Straftaten verurteilt worden sind und bei denen weitere Wiederholungen zu befürchten sind, durch therapeutische Mittel und soziale Hilfe ermöglichen, neue Einsichten $\mathrm{zu}$ gewinnen und sich neue Formen der Lebensbewältigung anzueignen" (Arbeitskreis Sozialtherapeutische Anstalten im Justizvollzug e. V. 2016, S. 37).

Es entstanden 1969 zunächst 2 Modellanstalten und daraufhin weitere Einrichtungen, um das Inkrafttreten des Gesetzes vorzubereiten. Auch wenn seitdem die Anzahl der sozialtherapeutischen Einrichtungen stets zunahm, trat statt der Maßregellösung das Strafvollzugsgesetz (StVollG) in Kraft, das im Rahmen einer sogenannten Kann-Bestimmung ermöglichte, einen Gefangenen in eine sozialtherapeutische Einrichtung zu verlegen, ,wenn die besonderen therapeutischen Mittel und sozialen Hilfen einer solchen Anstalt zu seiner Resozialisierung angezeigt sind“ (§ 9 StVollzG). Einen großen Einfluss auf die Entwicklung der Sozialtherapie hatte das „Gesetz zur Bekämpfung von Sexualdelikten und anderen gefährlichen Straftaten“ im Jahr 1998. Dabei wurde die bis dato freiwillige Aufnahme in die Sozialtherapie durch eine verpflichtende Aufnahme gemäß $\S 9$ Abs. 1 StVollzG ergänzt, und für die Personen, die wegen eines Sexualdelikts zu mehr als 2 Jahren Freiheitsstrafe verurteilt worden waren, wurde die Verlegung in eine sozialtherapeutische Einrichtung gesetzlich verpflichtend geregelt.

Um eine Einheitlichkeit in der Sozialtherapie für die anwachsende Anzahl von Einrichtungen anzustreben, formulierte der 1983 gegründete Arbeitskreis Sozialtherapeutische Anstalten e. V. (1988) Mindeststandards für die Ausgestaltung sozialtherapeutischer Einrichtungen. Diese wurden fortlaufend überarbeitet und aktualisiert (Arbeitskreis Sozialtherapeutische Anstalten im Justizvollzug e. V. 1988, 2001, 2016; Wischka und Specht 2001). Diese Mindeststandards dienen der Qualitätssicherung und betreffen die Bereiche Größe und Ausstattung der Einrichtung, räumliche Gegebenheiten, Angebote, personelle Ausstattung sowie Hinweise zu Indikation und Planung des Behandlungsrahmens. Ebenfalls empfohlen wurde durch den Arbeitskreis die Durchführung einer regelmäßigen Stichtagserhebung zu Gefangenen, Personal und weiteren Gegebenheiten in den sozialtherapeutischen Einrichtungen. Diese Stich- tagserhebung wird seit 1997 von der Kriminologischen Zentralstelle (KrimZ) durchgeführt, weshalb jedes Jahr die entsprechenden Informationen bei allen sozialtherapeutischen Einrichtungen in Deutschland zum 31. März abgefragt werden. Die erhobenen Daten, die zwischen 1997 und 2019 für 23 Jahrgänge erfasst wurden, werden im Folgenden hinsichtlich ihrer längsschnittlichen Trends und Entwicklungen dargestellt und diskutiert.

\section{Methode}

\section{Untersuchungsablauf}

Zur Datenerhebung wird der Erhebungsbogen zum 31. März eines jeden Jahres an alle in Deutschland aktiven sozialtherapeutischen Einrichtungen im Justizvollzug verschickt, wobei zuvor Änderungen hinsichtlich der Anzahl bei den 16 Bundesländer abgefragt werden. Ausgefüllt wurden die Erhebungsbogen von allen aktiven sozialtherapeutischen Einrichtungen im jeweiligen Erhebungsjahr. Bearbeiter des Fragebogens sind Angestellte der sozialtherapeutischen Einrichtungen, zumeist die Leitung, der psychologische oder der (sozial-)pädagogische Fachdienst, z. T. mit Unterstützung von anderen Fachdiensten oder der Personalabteilung. Die Rücksendung des Bogens ist bis zum 30. April eines jeden Erhebungsjahres terminiert. Anschließend werden die jeweiligen Bearbeiterinnen und Bearbeiter in den sozialtherapeutischen Einrichtungen kontaktiert, falls unklare, unvollständige oder widersprüchliche Antworten gemeldet wurden. Die Ergebnisse werden elektronisch ausgewertet und als elektronischer Bericht jährlich auf der Homepage der KrimZ (https://www.krimz.de/publikationen/texte.html) veröffentlicht.

\section{Datenbasis und statistische Auswertung}

Bei der KrimZ-Stichtagserhebung zur Sozialtherapie handelt es sich folglich um eine Vollerhebung, d.h., alle aktiven sozialtherapeutischen Einrichtungen stellen im jeweiligen Berichtsjahr die abgefragten Informationen bereit. Im Jahr 2019 gaben zwei Einrichtungen an, die sozialtherapeutische Einrichtung nicht (mehr) aktiv zu betreiben, d.h. kein Personal zu beschäftigen und/oder keine inhaftierten Personen dort zu betreuen, sodass diese, bis auf die allgemeine Darstellung der Anzahl der sozialtherapeutischen Einrichtungen, aus den weiteren Statistiken ausgenommen wurden, um Verzerrungen in den deskriptiven Statistiken zu vermeiden.

Die jährlichen Erhebungsbogen umfassen Fragen zur Anzahl der sozialtherapeutischen Einrichtungen, über die Demografie von den Gefangenen, über institutionelle Vorgänge und die personelle Ausstattung. Die Fragen unter- 
Abb. 1 Anzahl der sozialtherapeutischen Einrichtungen insgesamt und des Jugendstrafvollzugs (JVZ) zwischen 1969 und 2019

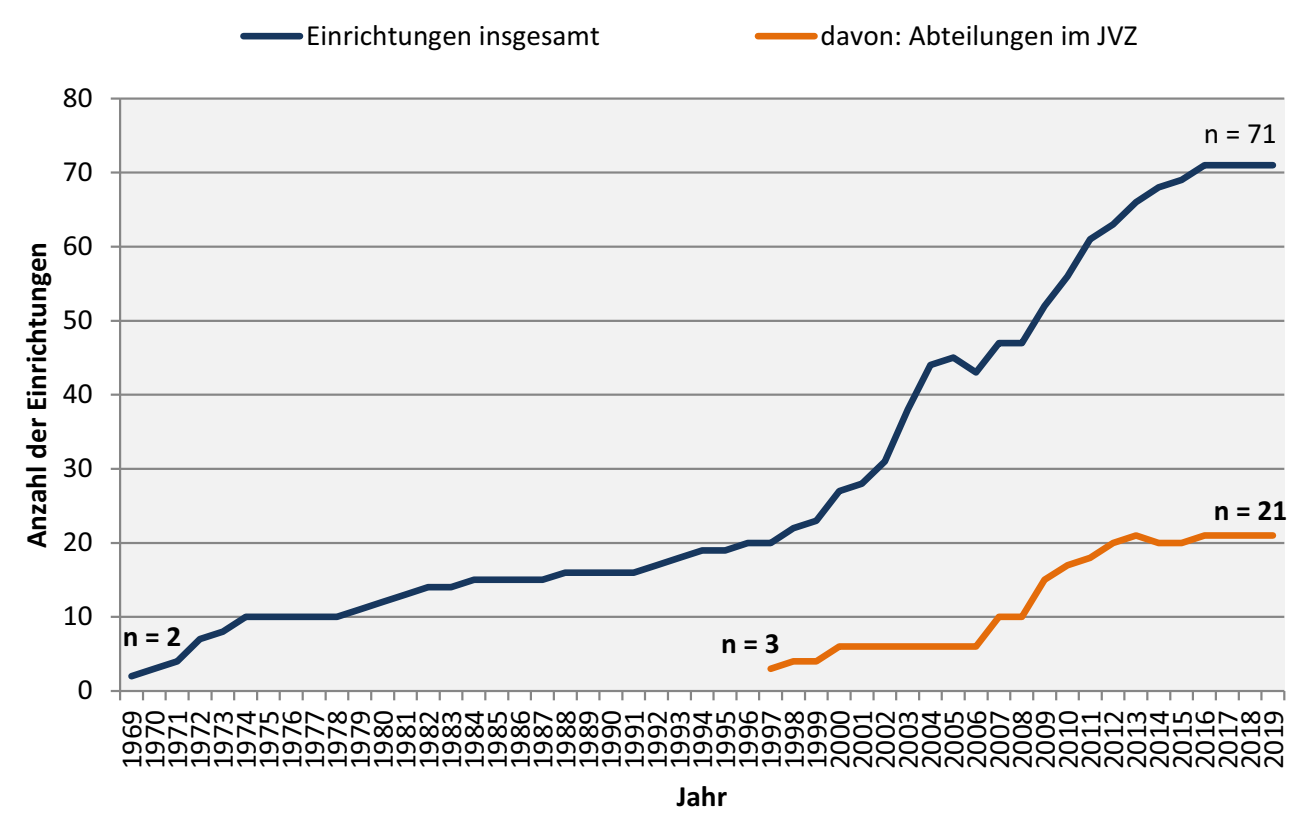

lagen über die Jahre hinweg kleineren Veränderungen und Anpassungen (sämtliche Fragebogen sind in den Stichtagsberichten abgedruckt und können elektronisch unter https://www.krimz.de/publikationen/texte.html abgerufen werden). Trotz dieser eher marginalen Änderungen können so Aussagen über die Anzahl der sozialtherapeutischen Einrichtungen, über die Entwicklung der Belegungszahlen, über institutionelle Vorgänge und das Personal über die vergangenen 23 Jahre hinweg getroffen und längsschnittlich analysiert werden. Da die Fragen des Erhebungsbogens zumeist aggregierte Informationen über die Gegebenheiten in sozialtherapeutischen Einrichtungen enthalten (z.B. wie viele Gefangene waren zum Stichtag zwischen 18 und 21 Jahre alt) und die Erhebung eine Vollerhebung darstellt, wurden die Angaben der Einrichtungen zumeist auf deskriptiv statistischer Ebene anhand von Prozentangaben oder Verlaufsdarstellungen in absoluten Zahlen ausgewertet.

\section{Ergebnisse}

\section{Versorgungslage durch sozialtherapeutische Einrichtungen}

Der Beginn der sozialtherapeutischen Behandlung im deutschen Justizvollzug liegt im Jahr 1969, in dem im Zuge der großen Strafrechtsreform „die Unterbringung in einer sozialtherapeutischen Anstalt" eine Maßregel der Besserung und Sicherung werden sollte ( $\$ 65$ StGB a.F.). Es wurden zwei Modellanstalten eröffnet, zum einen in HamburgBergedorf und zum anderen auf dem Hohenasperg (BadenWürttemberg). Bis 1981 wurden weitere 10 sozialtherapeu- tische Einrichtungen eröffnet, um das Inkrafttreten des Reformgesetzes vorzubereiten (Abb. 1). Einige dieser Modellbzw. Erprobungsanstalten wurden wissenschaftlich begleitet, um die Wirksamkeit der sozialtherapeutischen Einrichtungen auf die Rückfallwahrscheinlichkeit der Gefangenen zu untersuchen (Egg 2010). Auch wenn 1977 im StVollzG mit $\S 9$ eine Rechtsgrundlage für die Verlegung in eine sozialtherapeutische Einrichtung geschaffen wurde, so schrieb diese keine Verlegung vor, sondern ermöglichte sie lediglich, weshalb sie einen eher leichten Anstieg der Anzahl sozialtherapeutischer Einrichtungen nach sich zog.

Das Aufheben des $§ 65$ StGB a.F. zu Beginn 1985 ging mit einer Stagnation des Ausbaus sozialtherapeutischer Einrichtungen einher, die erst mit dem Gesetz zur Bekämpfung von Sexualdelikten und anderen gefährlichen Straftaten 1998 endete. Mit diesem Gesetz wurde in § 9 I StVollzG geregelt, dass wegen bestimmter Sexualdelikte zu einer über 2-jährigen Freiheitsstrafe verurteilte Personen auch ohne ihre Einwilligung in eine sozialtherapeutische Einrichtung verlegt werden sollten. Ab 1998 ist deshalb ein starker Anstieg der Einrichtungsanzahl zu verzeichnen, der ab 2003 besonders drastisch ausfällt, da die Verlegung nach § 9 I StVollzG von einer „Soll-Bestimmung“ zu einer „IstBestimmung" geändert wurde.

Die Anzahl aller Einrichtungen erreichte erst 2016 einen Sättigungspunkt und blieb seitdem bis zum Stichtag 2019 stabil bei 71 Einrichtungen, wobei 2 Einrichtungen - wie bereits oben dargestellt - im Berichtsjahr 2019 angegeben hatten, dass sie den Betrieb bis auf Weiteres eingestellt hatten.

Sozialtherapeutische Einrichtungen für Verurteilte nach Jugendstrafrecht gab es erst ab dem Jahr 1996. Ab 2007 wurden diese beträchtlich ausgebaut, als mit der Föderalis- 
Abb. 2 Freie und belegte Haftplätze in sozialtherapeutischen Einrichtungen zwischen 1997 und 2019

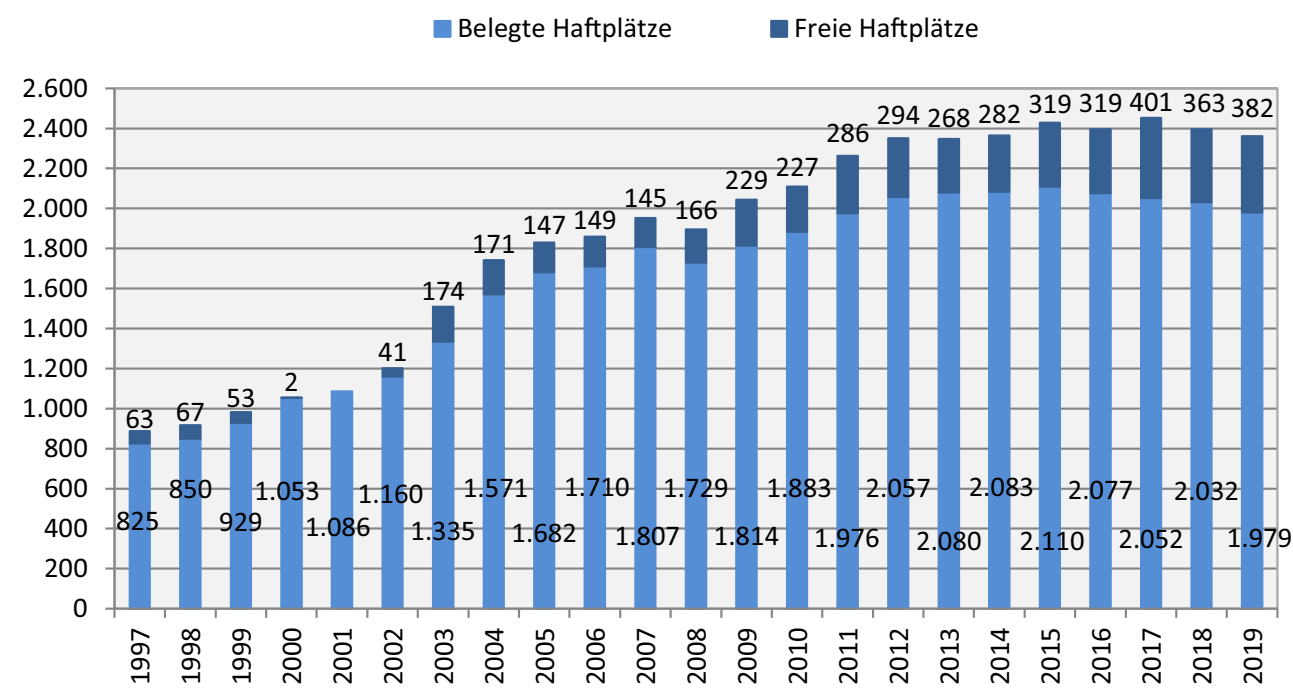

musreform der Jugendvollzug 2008 durch die Länder auf eine eigene gesetzliche Grundlage gestellt wurde und die Versorgung in diesem Bereich ausgebaut werden musste. Auch diese Zahl näherte sich seit 2012 einer Sättigungsgrenze an. Heute existieren 21 Einrichtungen, die speziell für Verurteilte nach Jugendstrafrecht zuständig sind. Das Gesetz zur bundesrechtlichen Umsetzung des Abstandsgebotes im Recht der Sicherungsverwahrung, das 2013 in Kraft trat, hatte, abgesehen von der Einrichtung in Werl, die ausschließlich für Sicherungsverwahrte zuständig ist, bislang keine Auswirkung auf die Ausgestaltung der Einrichtungen. Insgesamt wurden im Jahr 201971 Einrichtungen gezählt, wovon 21 für nach Jugendstrafrecht Verurteilte und 6 für Frauen zuständig waren. Hierbei handelt es sich um $62 \mathrm{Ab}$ teilungen, 6 selbstständige Anstalten und 3 sonstige Einrichtungen (Außenstellen, Teilanstalt oder Teilabteilung).

Die verfügbaren Plätze in den sozialtherapeutischen Einrichtungen wurden im Rahmen der Stichtagserhebung seit 1997 erfasst (Abb. 2). Während 1997 noch 888 Plätze mit einer Belegungsquote von $93 \%$ verfügbar waren, waren es 20172453 Plätze, von denen jedoch lediglich $84 \%$ belegt waren. In den letzten 2 Jahren zeichnet sich ein leicht rückläufiger Trend ab; im Jahr 2019 wurden 2361 Haftplätze gezählt. Die Belegungsquote sinkt bereits seit 2007 stetig und erreichte mit $84 \% 10$ Jahre später ihren Tiefstand. Der Anteil der Plätze im offenen Vollzug betrug zu Beginn der Stichtagserhebung 12,5\%, erreichte ein Jahr später seinen Höchststand mit 16,6\% und schwankt seit 2006 konstant um einen Mittelwert von etwa 3\%, wobei manche Einrichtungen angaben, dass weitere Plätze im offenen Vollzug bei Bedarf bereitgestellt werden könnten. Der Anteil belegter Plätze im offenen Vollzug, relativ zu allen belegten Plätzen, liegt im Mittel zwischen 2006 und 2019 ebenfalls bei 2,8\%.

Die Analyse der räumlichen Ausstattung seit 2011 ergibt, dass durchschnittlich $66 \%$ der Wohngruppen $\leq 12$ Plätze und dementsprechend $34 \%$ Wohngruppen $>12$ verfügbare Plätze/Wohngruppe aufweisen. Diese Bedingungen verbessern sich insofern, als dass in den letzten 2 Jahren erstmals $\geq 70 \%$ der Wohngruppen $\leq 12$ Plätze aufweisen und damit die Empfehlungen der Arbeitskreises Sozialtherapie (2016) einhalten.

\section{Die Gefangenen}

Die Gefangenen, die in den sozialtherapeutischen Einrichtungen aufgenommen wurden, waren im Jahr 1997 noch hauptsächlich zwischen 21 und 40 Jahre alt, also eher den jüngeren Erwachsenen zuzuordnen. Sehr junge Gefangene, die jünger als 21 Jahre alt waren, bzw. ältere Gefangene, die über 50 Jahre alt waren, machten zu diesem Zeitpunkt nur jeweils eine Minderheit in den sozialtherapeutischen Einrichtungen aus. Diese Altersverteilung änderte sich über die Jahre insofern, als dass im Jahr 2019 alle Altersgruppen zu vergleichbaren Anteilen vertreten sind (Abb. 3) - ausgenommen die Personen, die unter 21 Jahre alt sind, die weiterhin mit 10\% die Minderheit darstellen. Bemerkenswert ist hierbei, dass die Gruppe der über 50-jährigen Inhaftierten seit 2017 erstmals die größte Altersgruppe darstellt.

Seit 2011 wurde in der Stichtagserhebung erfasst, wie hoch der Anteil der deutschen bzw. nichtdeutschen Gefangenen in sozialtherapeutischen Einrichtungen ausfällt, auch in Abhängigkeit von der jeweiligen Altersstruktur. Die Quoten, die im Jahr $201192 \%$ Deutsche bzw. 8\% Nichtdeutsche umfassten, blieben bis 2019, als $88 \%$ Deutsche und $12 \%$ Nichtdeutsche erfasst wurden, relativ stabil, hinsichtlich der Nichtdeutschen auf niedrigem Niveau.

Die Dauer der in der Bezugssache verhängten Freiheitsoder Jugendstrafe wurde ebenfalls seit 1997 jährlich erfasst (Abb. 4). Es wird ersichtlich, dass zu Beginn der Aufzeichnungen besonders viele Personen mit 5- bis 7-jähri- 
Abb. 3 Altersgruppen in sozialtherapeutischen Einrichtungen zwischen 1997 und 2019
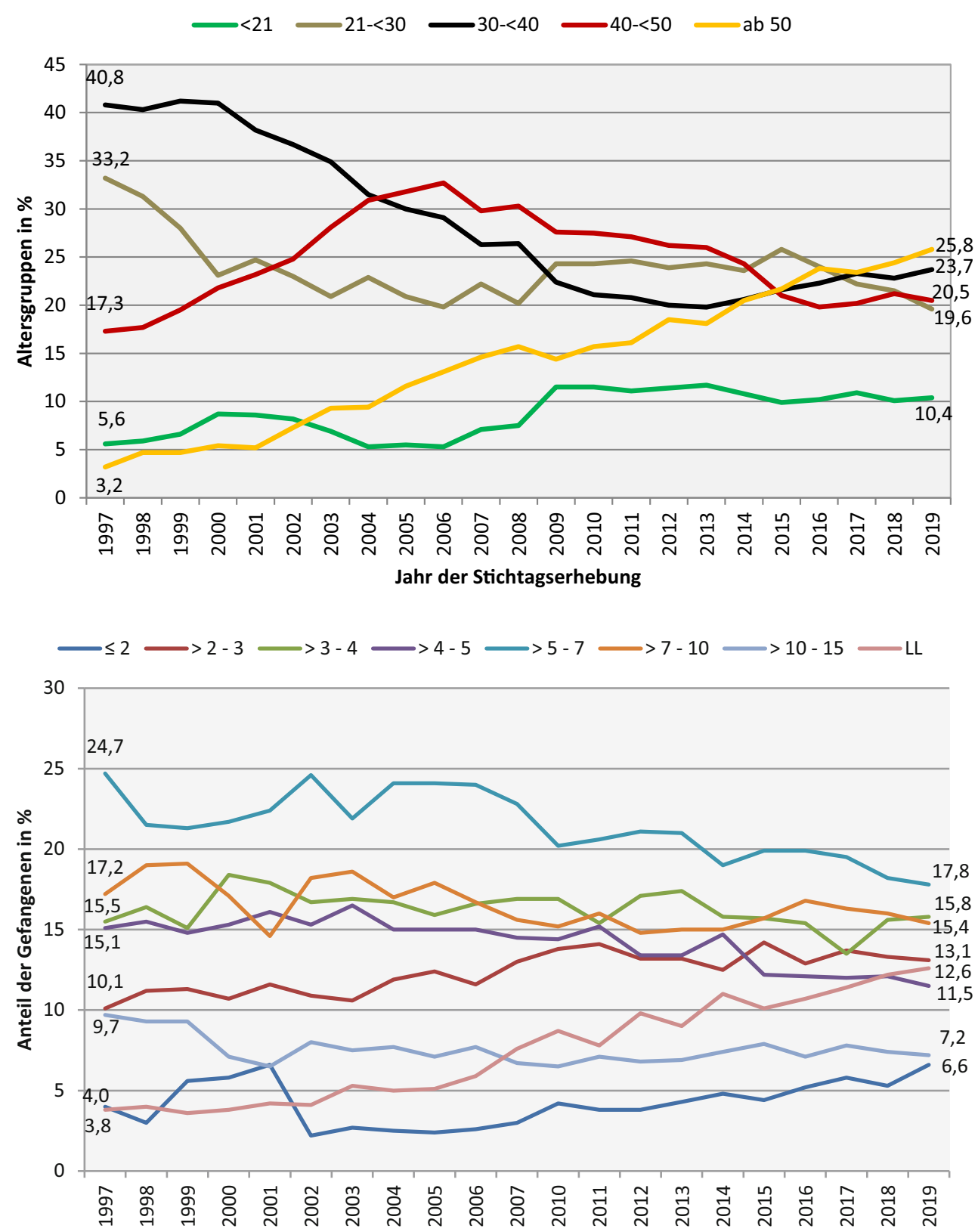

Abb. 4 Dauer der Freiheitsbzw. Jugendstrafe der Gefangenen zwischen 1997 und 2019 gen Freiheitsstrafen in sozialtherapeutische Einrichtungen aufgenommen wurden und der Teil derer mit $\leq 2$ Jahren bzw. lebenslanger Freiheitsstrafe die Minderheit ausmachte. Dies änderte sich über die Jahre insofern, als dass der Anteil der Personen mit einer Strafdauer zwischen 5 und 7 Jahren sichtbar ab- und dafür besonders der Anteil mit lebenslangen Freiheitsstrafen deutlich zunahm. Die Anteile anderer Personengruppen blieben innerhalb kleinerer lokaler Schwankungen einigermaßen konstant.

Seit Beginn der Stichtagserhebungen wurde erfasst, ob bei den Gefangenen auch zusätzlich eine Maßregel nach $\S \S 63,64,66$ bzw. 66a StGB angeordnet bzw. vorbehalten wurde. Es zeigt sich, dass dieser Anteil über die Jahre hinweg anstieg, von 2,8\% im Jahr 1997 bis 10,4\% im Jahr
2019. Seit 2011 wurde getrennt erfasst, welche der Maßregeln im Einzelnen angeordnet oder vorbehalten waren, und es zeigte sich, dass die Sicherungsverwahrung den höchsten Anteil der Maßregeln darstellt, und zwar von $2011 \mathrm{mit}$ 7,0\% bis 2019 mit 8,6\% an allen Gefangenen der Einrichtungen. Der Anteil der Personen in den sozialtherapeutischen Einrichtungen, die sich in der Unterbringung der Sicherungsverwahrung befanden, bewegte sich seit 2011 zwischen 3,0\% und 3,8\%.

Die Anzahl der Vorstrafen der Gefangenen wurde seit 1998 jährlich in Form von Kategorien erfasst. Hierbei ist ersichtlich, dass sich die prozentuale Aufteilung seit Beginn der Erhebung nicht stark veränderte und nur leichte Schwankungen zu beobachten waren. Betrachtet man 
Abb. 5 Schwerste Straftat in der Bezugssache der Insassen in sozialtherapeutischen Einrichtungen zwischen 1997 und 2019

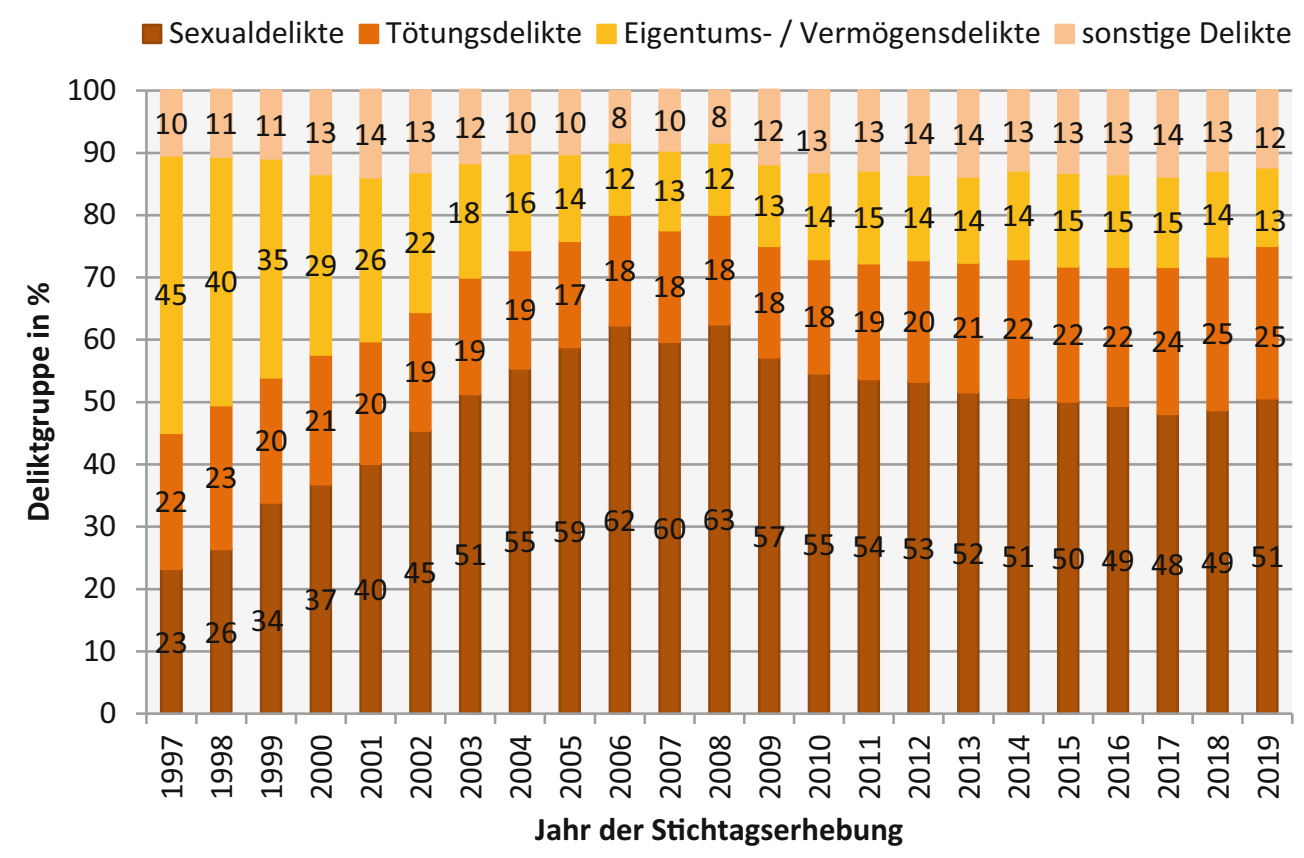

diese Mittelwerte über die Jahre hinweg, so ergibt sich, dass Personen ohne Vorstrafenbelastung im Mittel $40 \%$ (Min. $=35 \%$, Max. = 44\%) ausmachten, der Anteil von Personen mit geringer strafrechtlicher Vorbelastung (1-2 Vorstrafen) im Mittel bei $29 \%$ lag (Min.=25\%, Max.=29\%), während der Anteil an Personen mit hoher Vorstrafenbelastung (9-15 Vordelikte) bei 8\% (Min.=7\%, Max.=10\%) lag.

Eine wichtige Information betrifft die schwerste Straftat der Bezugssache, also den schwersten Straftatbestand, der Gegenstand der aktuellen Inhaftierung bzw. der Anordnung oder dem Vorbehalt der Sicherungsverwahrung war. Durch das „,Gesetz zur Bekämpfung von Sexualdelikten und anderen gefährlichen Straftaten“"von 1998 war zu erwarten, dass insbesondere der Teil der Straftäter besonders hoch war, der wegen eines Sexualdelikts zu einer Freiheits- oder Jugendstrafe verurteilt wurde. Die Anteile der jeweils schwersten Straftaten aller Gefangenen seit 1997 bis 2019 zeigt Abb. 5. Hierbei ist seit 1997 ein Anstieg des Anteils der Sexualstraftäter zu verzeichnen, der 2008 den Höhepunkt erreicht und seitdem langsam sinkt, hauptsächlich zugunsten einer sukzessive höheren Quote von Tötungsdelikten. Eigentumsund Vermögensdelikte, die zu Beginn der Erhebungen fast die Hälfte der inhaftierten Personen ausmachten, spielen heute mit knapp $13 \%$ eine eher untergeordnete Rolle.

Da die Sexualstraftäter seit 2003 bis heute mindestens knapp $50 \%$ bis über $60 \%$ aller Gefangenen darstellen, wurden für diese Gruppe detailliertere Analysen vorgenommen. Die Abb. 6 zeigt, dass seit den ersten Jahren der Stichtagserhebung besonders viele Gefangene wegen sexuellem Missbrauchs von Kindern inhaftiert waren und heute mit knapp $60 \%$ die Mehrheit der Sexualstraftäter/-innen stellt; ein gu- tes Drittel (aktuell etwa 35\%) sind Personen, die aufgrund sexueller Nötigung oder Vergewaltigung verurteilt wurden, während die Kategorie der sonstigen Sexualdelikte konstant niedrig blieb (ca. $8 \%$ ).

\section{Institutionelle Vorgänge}

Um die Belegungsprozesse in den sozialtherapeutischen Einrichtungen quantifizieren zu können, wurde erfasst, wie viele Personen im Berichtsjahr aufgenommen wurden und wie viele Personen innerhalb eines Jahres abgingen, inklusive der hierfür genannten Gründe. Die jährlichen Aufnahmen und Abgänge (Entlassung, Rückverlegung durch Anstalt, Rückverlegung auf Antrag des Gefangenen, Verlegung nach Plan und sonstiger Abgang), jeweils prozentual bestimmt an allen Abgängen in diesem Jahr, zeigt Abb. 7 . Besonders viele Aufnahmen wurden in den Jahren verzeichnet, in denen viele neue Plätze geschaffen wurden, insbesondere um das Jahr 2004. Eine Entlassung in Freiheit ist mit ca. $48 \%$ zu jedem Berichtsjahr der Hauptgrund eines Abgangs, gefolgt von der Rückverlegung durch die Anstalt, die im Mittel $30 \%$ beträgt. Eine eher untergeordnete Rolle spielten hingegen die Rückverlegung auf Antrag des Gefangenen $(7,5 \%)$, die Verlegung nach Plan (10,6\%; z. B. in eine andere Einrichtung) und die sonstigen Abgänge (4,0\%; z.B. aufgrund von Abschiebung oder Suizid).

Die sozialtherapeutischen Einrichtungen bieten grundsätzlich gemäß $\S 125$ f StVollzG die Wiederaufnahme eines früheren Gefangenen auf eigenen Antrag hin an. Die Zahl der wiederaufgenommenen früheren Gefangenen ist durchweg niedrig und bewegte sich in den letzten 23 Jahren zwischen 1 (2003) und 29 (2010) Personen/Jahr. Die nach- 
Abb. 6 Schwerste Straftat in der Bezugssache bei sexuell motivierten Straftaten zwischen 1997 und 2019
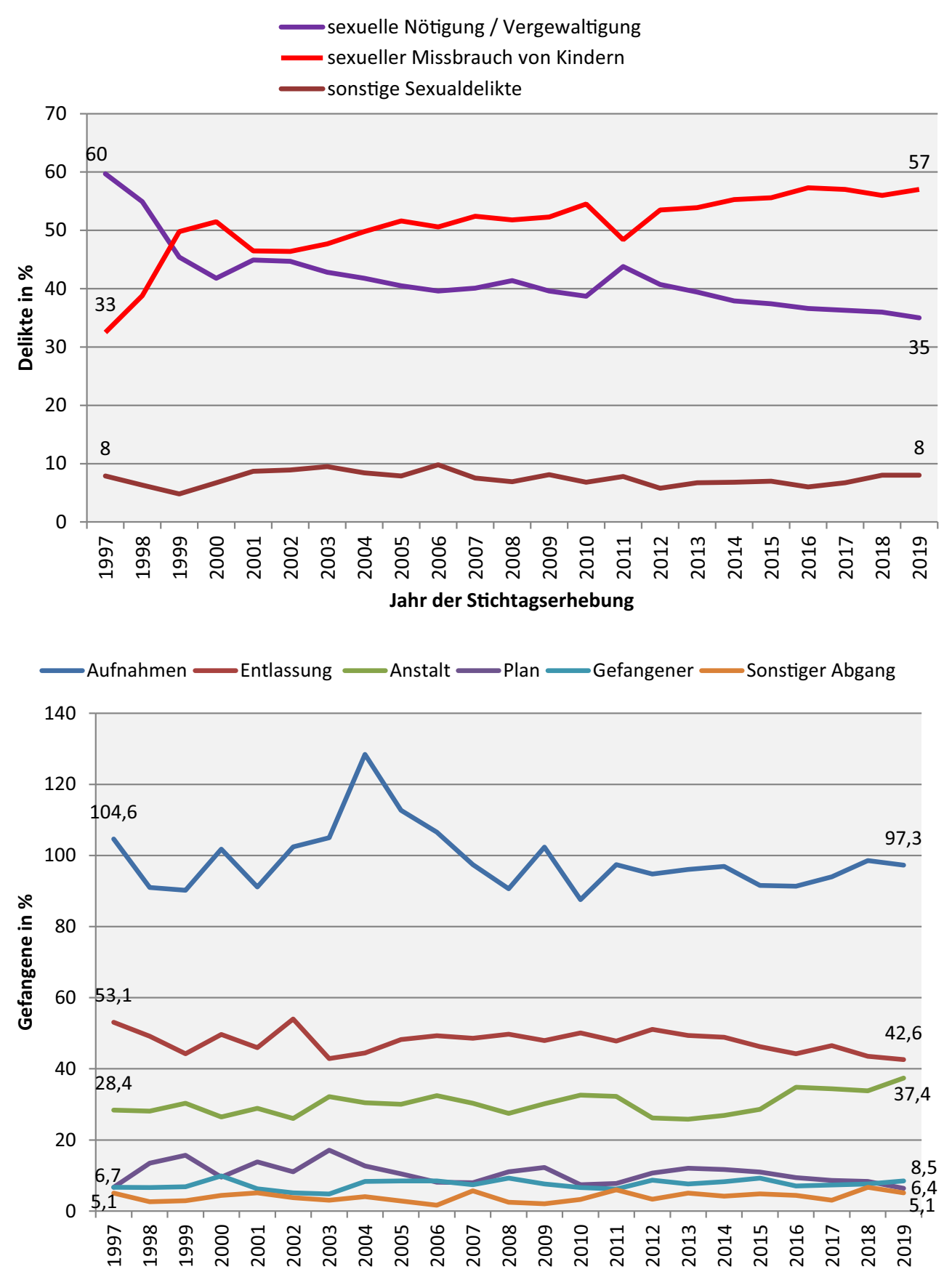

Abb. 7 Aufnahmen und Gründe für Abgänge (Entlassung in Freiheit, Rückverlegung durch Anstalt, Verlegung nach Plan, Rückverlegung auf Antrag des Gefangenen und sonstiger $\mathrm{Ab}$ gang) gehende Betreuung gemäß $§ 126$ StVollzG soll ebenfalls durch die SothEn gewährleistet werden. Hiervon machten pro Jahr immer mehr Gefangene Gebrauch: Während es 1997 noch 27 Gefangene waren, stieg dies bis ins Jahr 2016 konstant auf 406 betreute Personen an und ging bis 2019 auf 275 Personen zurück.

Die Lockerungsstufe eines jeden Gefangenen wurde mittels einfacher Auszählung in jedem Berichtsjahr erfasst. Wie sich die höchste Lockerungsstufe aller Gefangenen über die Zeit hinweg entwickelte, zeigt Abb. 8. Es wird deutlich, dass der prozentuale Anteil derjenigen ohne $\mathrm{Zu}$ - lassung von Ausführungen über die Zeit hinweg angestiegen ist, v.a. auf Kosten des Freigangs und Sonderurlaubs, wobei auch der Anteil derjenigen, die Ausgang oder Regelurlaub genehmigt bekommen hatten, bis 2006 abgenommen und sich seither unterhalb der 20\%-Marke befand.

\section{Das Personal}

Die Anzahl der Personalstellen stieg mit der Anzahl der eingerichteten Haftplätze. Im Rahmen der Stichtagserhebung war allerdings entscheidender, wie hoch die Anzahl 
Abb. 8 Höchste Lockerungsstufe der Gefangenen zum Stichtag zwischen 1997 und 2019

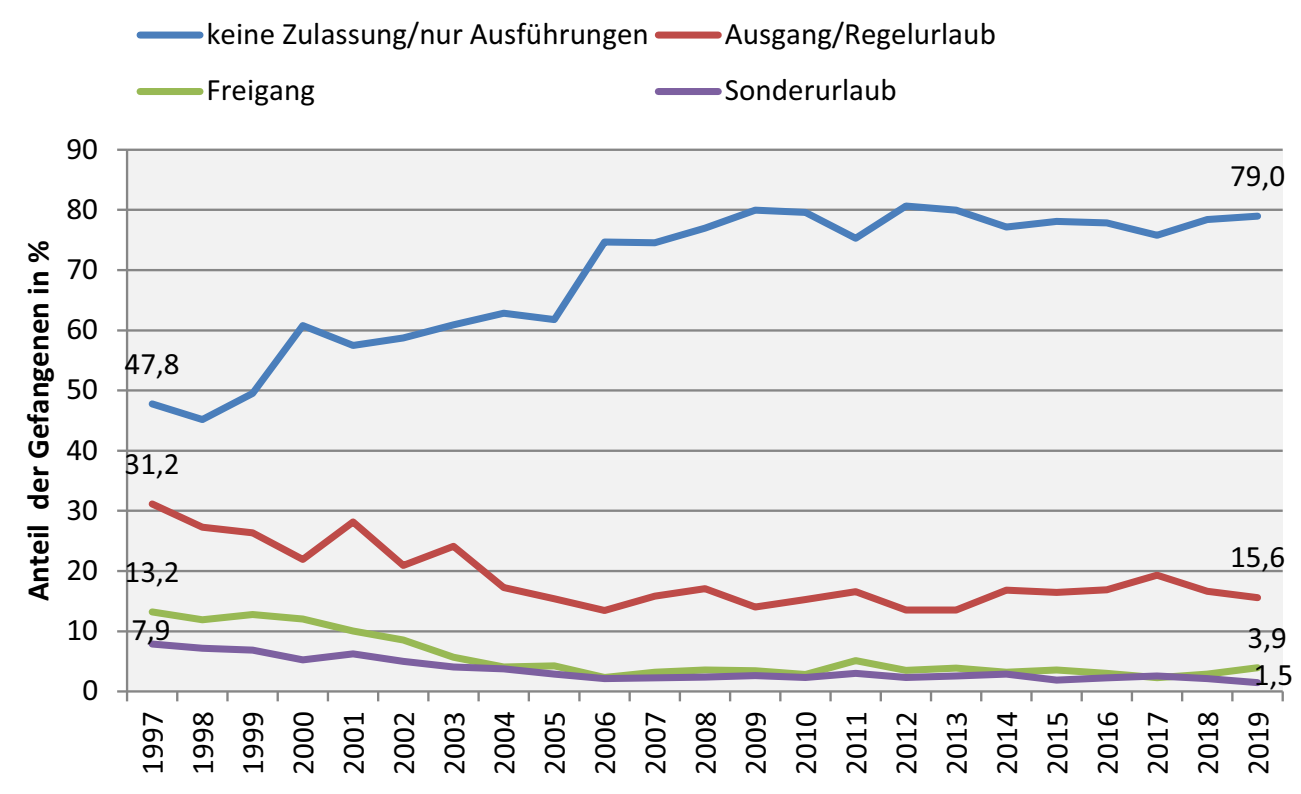

Abb.9 Anzahl der Haftplätze pro Personalstelle, Fachdienst (FD) oder allgemeiner Vollzugsdienst/ Werkdienststelle (AVD/WD) zwischen 1997 und 2019

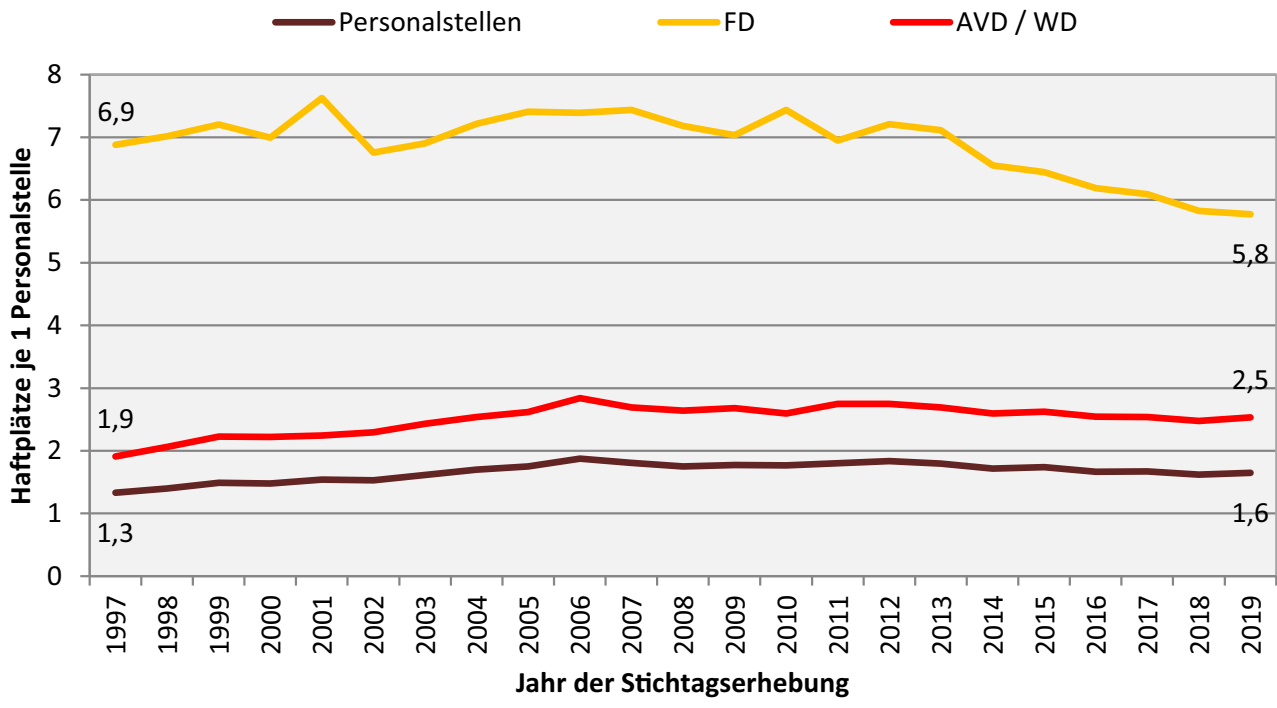

der Haftplätze pro Personalstelle ist, als Maß für die Versorgungslage durch das Personal. Abb. 9 zeigt die Anzahl der Haftplätze pro Personalstelle, pro allgemeine Vollzugsdienststellte (AVD) bzw. Werksdienststelle und pro Fachdienststelle. Es zeigt sich, dass sich die Anzahl der Haftplätze pro AVD/Werkdienststelle über die Jahre hinweg leicht erhöhte und 2019 bei 2,5 Haftplätzen lag. Dagegen sank der Anteil der Haftplätze, für die jeweils ein Fachdienst verantwortlich war, über die Jahre von 6,9 bis auf 5,8 in 2019. Insgesamt verschlechterte sich der Personalschlüssel über die Jahre minimal, da 1997 noch 1,3 Haftplätze auf eine Personalstelle und 2019 1,7 Haftplätze auf eine Personalstelle fielen. Dennoch zeigt der Trend, dass tendenziell mehr Personalstellen in den Fachdiensten geschaffen wurden und dafür weniger AVD/Werkdienststellen pro Haftplatz zur Verfügung standen.
Betrachtet man den Anteil der Frauen im Personal sozialtherapeutischer Einrichtungen, so zeigt sich, dass insbesondere in der Verwaltung der Frauenanteil am höchsten war und über die Jahre hinweg leicht angestiegen ist, auf 63,3\% (siehe Abb. 10). Besonders hoch war der Frauenanteil außerdem in den Fachdiensten, von $44 \%$ zu Beginn der Stichtagserhebung auf heute knapp $60 \%$. Ein geringerer Anteil von Frauen war beim AVD/Werkdienst festzustellen, wobei dieser von $17 \%$ in 1997 auf $21 \%$ in 2019 angestiegen war. Ein besonderer Verlauf ist für die Leitungspositionen zu beobachten, bei denen der Anteil an Frauen drastisch gestiegen war, von knapp $18 \%$ zu Beginn bis zu $53 \%$ bei der letzten Stichtagserhebung (Abb. 10).

Abschließend wurde erhoben, wie groß der Anteil der Supervision war, der durch die sozialtherapeutischen Einrichtungen angeboten wurde. Dass die Teamsupervision die 
Abb. 10 Anteil der Frauen in unterschiedlichen Personalbereichen zwischen 1997 und 2019

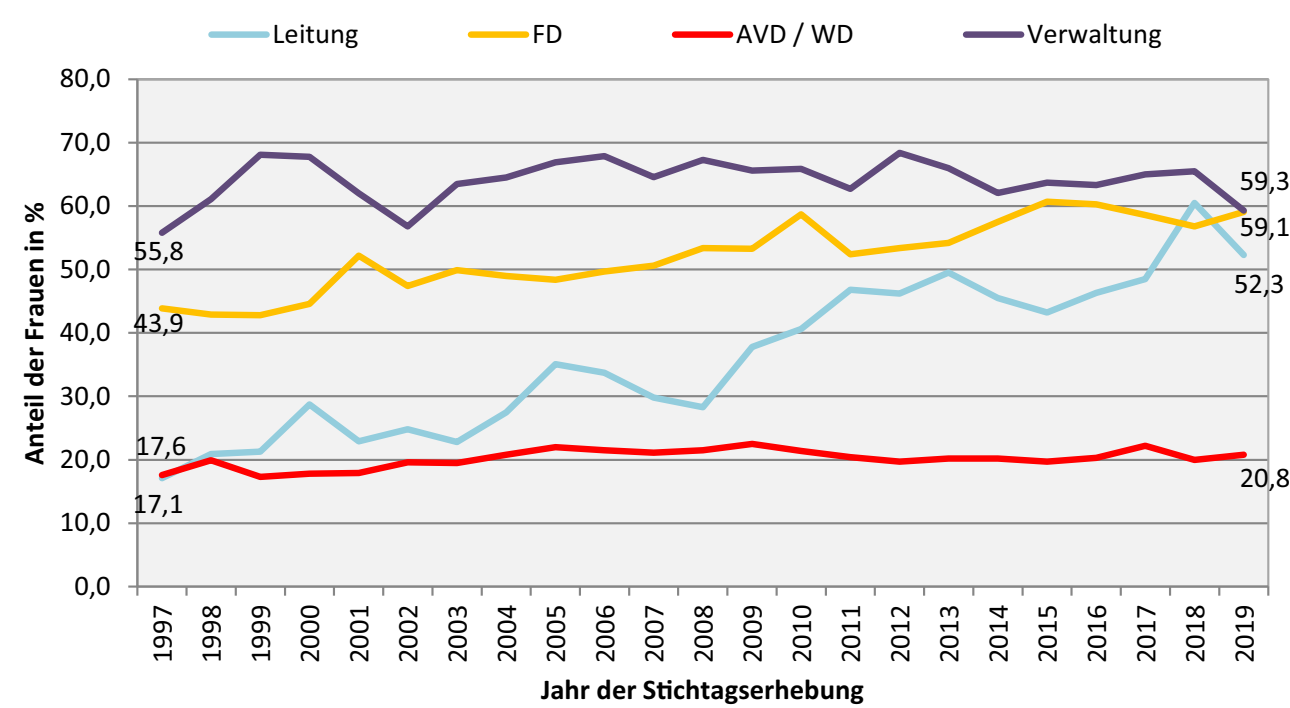

Abb. 11 Supervisionsformen in den sozialtherapeutischen Einrichtungen zwischen 1998 und 2019

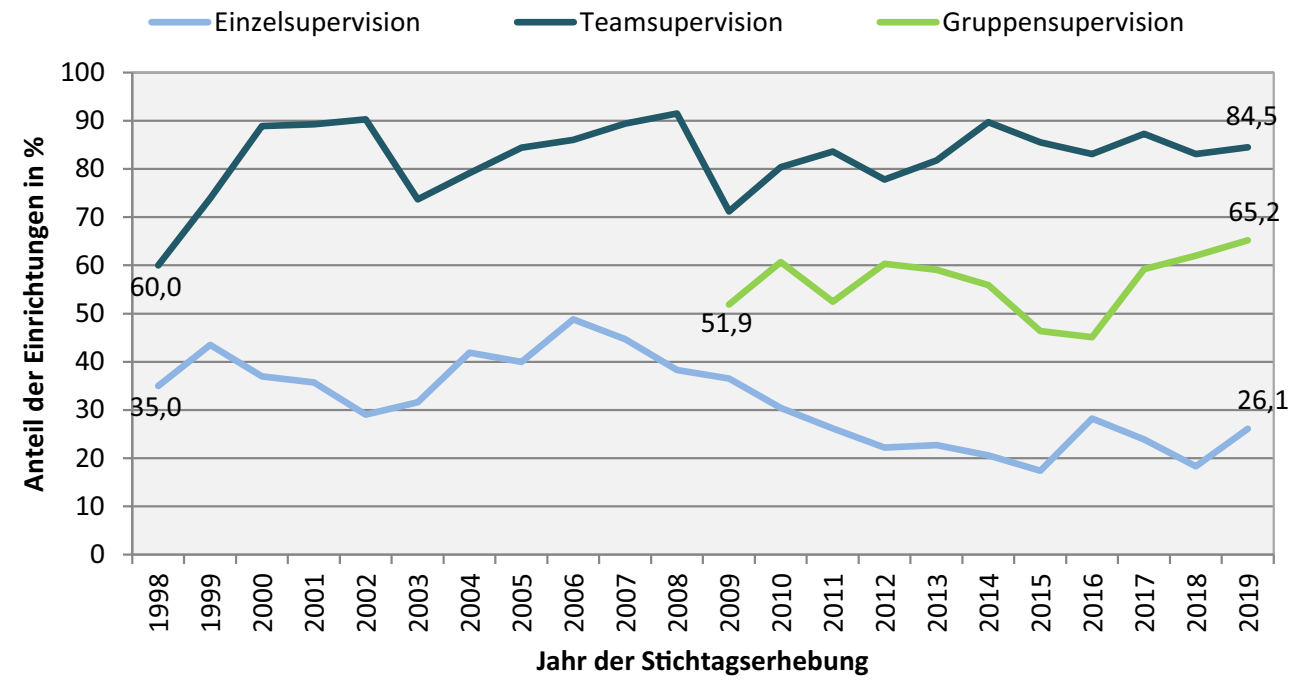

häufigste Art der Supervision ist und seit 1997 regelmäBig in vielen Einrichtungen eingesetzt wird, zeigt Abb. 11. Auch die Gruppensupervision, die seit 2009 erhoben wurde, wird von vielen Einrichtungen mit steigender Tendenz durchgeführt. Einzig die Einzelsupervision scheint etwas an Bedeutung zu verlieren.

\section{Diskussion}

Die vorliegenden Daten zur Stichtagserhebung zur Sozialtherapie zeigen, dass die Versorgungslage in Deutschland insbesondere seit 1998 stetig ausgebaut wurde. Ein maßgeblicher Grund hierfür lag und liegt an den gesellschaftspolitischen Forderungen, die schließlich Eingang in die rechts- und kriminalpolitischen Diskussionen gefunden haben, nach denen v.a. sexuell motivierte Straftaten gegen Kinder mit allen zur Verfügung stehenden staatlichen Mit- teln verhindert werden sollen (Kröber 1998). Dieser Trend zeigt sich zwar auch in anderen westlichen Jurisdiktionen, in Deutschland hielten sich jedoch repressive und therapeutische Anteile noch etwas mehr die Waage im Vergleich $\mathrm{zu}$ anderen Ländern in Europa oder dem angloamerikanischen Sprachraum (Rettenberger 2018). So ist es eine fast zwangsläufig zu erwartende Entwicklung, dass nach wie vor Personen, die aufgrund von Sexualdelikten (und hier insbesondere pädosexuellen Straftaten) verurteilt wurden, einen substanziellen Anteil an den Insassen sozialtherapeutischer Einrichtungen ausmachen. Gleichzeitig nimmt der Anteil von Personen, die aufgrund von Tötungsdelikten verurteilt wurden, stetig zu, während Personen, die aufgrund von (gewalttätigen) Eigentumsdelikten verurteilt wurden, einen rückläufigen Trend aufweisen.

Die Entwicklung der Altersstruktur straffällig gewordener Personen, die in sozialtherapeutischen Einrichtungen behandelt werden, spiegelt allgemeine demografische und 
versorgungspolitische Trends wieder: So wird wie in vielen Gesellschaftsbereichen auch im intramuralen Setting der Anteil von Personen, die sich in fortgeschrittenen Altersgruppen befinden, tendenziell höher, und es ist zu erwarten, dass diese Entwicklung nicht ab-, sondern in den kommenden Jahren eher weiterzunehmen wird. Damit ist eine Reihe von therapeutischen und vollzugspraktischen Implikationen verbunden, die in den kommenden Jahren (weiter-)diskutiert werden müssen. So stellen sich zwangsläufig bei einer zunehmend älter werdenden Behandlungsklientel einerseits intramurale (medizinische, psychotherapeutische, sozialarbeiterische) Versorgungsfragen, andererseits liegen hier oftmals besondere Herausforderungen für die extramurale (Entlass-)Situation vor (z. B. hinsichtlich betreuter Wohnheimplätze; zur aktuellen Praxis: z. B. Gregório Hertz et al. 2019). Beide Aspekte sind auch vor dem Hintergrund zu sehen, dass bei älter werdenden Inhaftiertengruppen nicht zwangsläufig von einer günstigeren Legalprognose auszugehen ist: Während manche Gruppen (z. B. nichtsexuell motivierte Gewaltstraftäter) mit zunehmenden Alter bessere Legalbewährungschancen besitzen, ist die Rückfallwahrscheinlichkeit bei sexuell motivierten Straftaten kaum vom fortschreitenden Lebensalter abhängig (z.B. Rettenberger et al. 2015; Wendt und Kröber 2009).

Aus der Beschreibung der in der Sozialtherapie untergebrachten Personen ergeben sich auch aus anderen Gründen Hinweise auf besondere therapeutische Herausforderungen: Wie frühere empirische Studien zur Sozialtherapieklientel zeigen konnten, handelt es sich bei in sozialtherapeutischen Einrichtungen untergebrachten Straffälligen in der Regel um Personen, die zumindest zu Beginn des Aufenthalts in der Sozialtherapie ein vergleichsweise hohes Rückfallrisiko und nur über relativ wenige Ressourcen verfügen (Brunner et al. 2016). Dadurch wird das Strafvollzugsziel, nämlich solche Personen mit speziellen Behandlungseinrichtungen zu erreichen, bei der von einem erhöhten Rückfallpotenzial ausgegangen werden muss, erreicht; gleichzeitig handelt es sich um eine besonders (heraus-)fordernde und intensive Aufgabe, die in den hier vorliegenden Ergebnissen u.a. darin zum Ausdruck kommt, dass nach wie vor eine vergleichsweise große Gruppe den Aufenthalt in der Sozialtherapie abbricht bzw. abbrechen muss. Aus früheren Untersuchungen ist bekannt, dass ein Behandlungsabbruch einerseits mit einem erhöhten Rückfallrisiko in Verbindung steht (Olver und Wong 2009, 2011), andererseits v. a. die Personen abbrechen, die die Behandlung aufgrund ihres Ausgangsrisikos und des (Fremd-)Gefährdungspotenzials am meisten benötigen würden (Brunner et al. 2019).

Aus therapeutischer Sicht ungünstig ist die Tendenz einer zunehmend restriktiveren Lockerungspraxis. Wie die Ergebnisse der vorliegenden Auswertung zeigen, nimmt der Anteil an Personen, die im Rahmen ihres Aufenthalts in der Sozialtherapie (weiterführende) Lockerungen erhalten, über die Jahre weiter ab und liegt stabil auf niedrigem Niveau. Für diese Entwicklung mag es sicherlich kriminalpolitisch nachvollziehbare Überlegungen geben; aus therapeutischer Sicht ist sie problematisch, da sozialtherapeutisch induzierte Veränderungen (wie bei den meisten übrigen therapeutischen Prozessen auch) die Möglichkeit benötigen, die angestoßenen Veränderungen zu erproben und so zu stabilisieren. Dieser Mangel an Erprobungsmöglichkeiten kann einen Behandlungsprozess erschweren und damit möglicherweise auch dazu beitragen, dass extramurale Behandlungsprogramme in der Vergangenheit tendenziell eine höhere Wirksamkeit erzielen als intramurale Therapieangebote (Lösel 2016; Schmucker und Lösel 2015).

Im Hinblick auf die personelle Ausstattung lassen die vorliegenden Daten auf eine zunehmende Professionalisierung der Sozialtherapie schließen, da der Anteil der unmittelbar in der therapeutischen Versorgung beteiligten Berufsgruppen stetig angewachsen ist. Diese zunehmende Professionalisierung wird durch Zusatzerhebungen bestätigt, die sich vorrangig mit der Eingangs-, Verlaufs- und Abschlussdiagnostik beschäftigten und die zeigen konnten, dass die Mehrheit der sozialtherapeutischen Einrichtungen nach (inter-)national üblichen Qualitätsstandards im Bereich der forensischen Psychotherapie und Psychologie arbeitet (Etzler und Rettenberger 2019).

Unabhängig von den genannten Punkten, die Hinweise für zukünftige Gestaltungsmöglichkeiten und -potenziale geben können, zeigen die vorliegenden Ergebnisse, dass sich in den letzten 23 Jahren viel getan hat, um die intramurale Versorgungslage in Deutschland zu verbessern, aber auch um die geforderten Mindeststandards für eine wissenschaftlich und klinisch fundierte Sozialtherapie zu erfüllen. Es scheint aktuell ein gewisser Sättigungseffekt feststellbar, sodass die angebotenen Plätze nicht weiterausgebaut werden und 2 Einrichtungen derzeit aufgrund des geringen Bedarfs inaktiv sind. Nach einer langen Phase des Aufbaus sozialtherapeutischer Einrichtungen scheint nun eine Phase der Konsolidierung, der Verbesserung und der Anpassung des Erreichten zu beobachten zu sein. Studien zur Wirksamkeit intramuraler Behandlungs- und Betreuungsprogramme (Lösel 2016; Schmucker und Lösel 2015) weisen generell auf einen signifikanten, wenn auch bislang als klein bis moderat ausgeprägten Wirksamkeitseffekt hin. Weitere Verbesserungen in den strukturellen Gegebenheiten, Weiterentwicklungen im Behandlungsangebot und der personellen Ausstattung sowie eine konsequente Implementierung wissenschaftlich fundierter Evaluationsstrukturen könnten dazu beitragen, die vielfach erreichten positiven Effekte in Zukunft noch weiterzusteigern.

Funding Open Access funding provided by Projekt DEAL.

Interessenkonflikt S. Etzler, M. Moosburner und M. Rettenberger geben an, dass kein Interessenkonflikt besteht. 
Open Access Dieser Artikel wird unter der Creative Commons Namensnennung 4.0 International Lizenz veröffentlicht, welche die Nutzung, Vervielfältigung, Bearbeitung, Verbreitung und Wiedergabe in jeglichem Medium und Format erlaubt, sofern Sie den/die ursprünglichen Autor(en) und die Quelle ordnungsgemäß nennen, einen Link zur Creative Commons Lizenz beifügen und angeben, ob Änderungen vorgenommen wurden.

Die in diesem Artikel enthaltenen Bilder und sonstiges Drittmaterial unterliegen ebenfalls der genannten Creative Commons Lizenz, sofern sich aus der Abbildungslegende nichts anderes ergibt. Sofern das betreffende Material nicht unter der genannten Creative Commons Lizenz steht und die betreffende Handlung nicht nach gesetzlichen Vorschriften erlaubt ist, ist für die oben aufgeführten Weiterverwendungen des Materials die Einwilligung des jeweiligen Rechteinhabers einzuholen.

Weitere Details zur Lizenz entnehmen Sie bitte der Lizenzinformation auf http://creativecommons.org/licenses/by/4.0/deed.de.

\section{Literatur}

Arbeitskreis Sozialtherapeutische Anstalten im Justizvollzug e.V. (1988) Mindestanforderungen an Sozialtherapeutische Einrichtungen. Monatsschr Kriminol Strafrechtsreform 71:334-335

Arbeitskreis Sozialtherapeutische Anstalten im Justizvollzug e.V. (2001) Mindestanforderungen an Sozialtherapeutische Einrichtungen. Forum Strafvollzug 50:40-41

Arbeitskreis Sozialtherapeutische Anstalten im Justizvollzug e.V. (2016) Sozialtherapeutische Anstalten und Abteilungen im Justizvollzug; Mindestanforderungen an Organisation und Ausstattung sowie Indikation zur Verlegung - Revidierte Empfehlungen des Arbeitskreises Sozialtherapeutische Anstalten im Justizvollzug e. V. - Stand 2016. Forum Strafvollzug 65:37-40

Brunner F, Neumann I, Yoon D, Rettenberger M, Stück E, Briken P (2019) Determinants of dropout from correctional offender treatment. Front Psychiatry 10:142. https://doi.org/10.3389/fpsyt. 2019.00142

Brunner F, Yoon D, Rettenberger M, Briken P (2016) Kriminologische und kriminalprognostische Merkmale der Insassen der Sozialtherapeutischen Anstalt Hamburg. Recht Psychiatr 34:221-227

Egg R (2010) Sozialtherapie: gestern, heute, morgen. In: Dölling D, Götting B, Meier B-D, Verrel T (Hrsg) Verbrechen - Strafe - Resozialisierung. DeGruyter, Berlin, S 313-336
Etzler S, Rettenberger M (2019) Psychologische Diagnostik im Rahmen der Behandlung von Gewalt- und Sexualstraftätern im Justizvollzug: Eine Vollerhebung diagnostischer Praxis der sozialtherapeutischen Einrichtungen in Deutschland. Diagnostica. https:// doi.org/10.1026/0012-1924/a000235

Gregório Hertz P, Breiling L, Turner D, Rettenberger M (2019) Die Praxis der ambulanten Nachsorge für haftentlassene Sexualstraftäter in Deutschland. Recht Psychiatr 37:157-164

Kröber HL (1998) Die Strafrechtsreformen zur Sexual- und Gewaltdelinquenz. Z Sex-Forsch 11:59-66

Lösel F (2016) Wie wirksam ist die Straftäterbehandlung im Justizvollzug? In: Rettenberger M, Dessecker A (Hrsg) Behandlung im Justizvollzug. Kriminologische Zentralstelle, Wiesbaden, S 17-52

Olver ME, Wong S (2009) Therapeutic responses of psychopathic sexual offenders: treatment attrition, therapeutic change, and longterm-recidivism. J Consult Clin Psychol 77:328-336. https://doi. org/10.1037/a0015001

Olver ME, Wong SCP (2011) Predictors of sex offender treatment dropout: psychopathy, sex offender risk, and responsivity implications. Psychol Crime Law 17:457-471. https://doi.org/10.1080/ 10683160903318876

Rettenberger M (2018) Unterstanding and managing risk of sexual offenders in Germany-a criminological success story or punitive hysteria? Sex Offender Treat 13:1-8

Rettenberger M, Briken P, Turner D, Eher R (2015) Sexual offender recidivism among a population-based prison sample. Int $\mathrm{J}$ Offender Ther Comp Criminol 59:424-444. https://doi.org/10.1177/ $0306624 X 13516732$

Schmucker M, Lösel F (2015) The effects of sexual offender treatment on recidivism: an international meta-analysis of sound quality evaluations. J Exp Criminol 11:597-630. https://doi.org/10. 1007/s11292-015-9241-z

Wendt F, Kröber HL (2009) Ältere Pädophile: Kein Rückgang der Delinquenz. Forens Psychiatr Psychol Kriminol 3:221-229

Wischka B, Specht F (2001) Integrative Sozialtherapie - Mindestanforderungen, Indikation und Wirkfaktoren. In: Rehn G, Wischka B, Lösel F, Walter M (Hrsg) Behandlung ,gefährlicher“ Straftäter. Grundlagen, Konzepte, Ergebnisse. Centaurus, Herbolzheim, S 249-263 